UC-20g

Issued: March 1984

\title{
The Hamiltonian Structure of Field-Line Equations
}

\author{
Pau! J. Channell
}

\section{DISCLAIMER}

This report was prepared as an account of work sponsored by an agency of the United States Government. Neither the United States Government nor any agency thereof, nor any of their employees, makes any warranty, express or implied, or assumes any legal liability or responsibility for the accuracy, completeness, or usefulness of any information, apparatus, product, or process disclosed, or represents that its use would not infringe privately owned rights. Reference herein to any specific commercial product, process, or service by trade name, trademark, manufacturer, or otherwise does not necessarily constitute or imply its endorsement, recommendation, or favoring by the United States Government or any agency thereof. The views and opinions of authors expressed herein do not necessarily state or reflect those of the United States Government or any agency thereof. 
THE HAMILTONIAN STRUCTURE OF FIELD-LINE EQUATIONS

by

Paul J. Channe il

\begin{abstract}
The canonical structure of the field-line equations in toroidal geometry is derived, and the global Hamiltonian for one choice of canonical coordinates is given explicitly.
\end{abstract}

Results from Hamiltonian perturbation theory, such as the KAM theorem ${ }^{1}$ and the existence of stochastic zones, 2 are of en used in discussing the behavior of magnetic field lines. However, the Hamiltonian structure is not evident, so these results are not obviously applicable. In this report I will disp!ay explicitly both the canonical structure of the equations and the Hamiltonian. Similar results, with the exception of the Hamiltonian, have been obtained previously by K. Symon.*

We are principally interested in toroidal configurations with nowhere vanishing toroidal field, a configuration of some practical interest, so we concentrate on this case. Thus, we use cylindrical coordinates, in which the field-line equations become

$$
\frac{\mathrm{dr}}{\mathrm{d} \vartheta}=\frac{\mathrm{r} \mathrm{B}_{\mathrm{r}}(\mathrm{r}, \mathrm{z}, \vartheta)}{\mathrm{B}_{\vartheta}(\mathrm{r}, z, v)}
$$

* These data were supplied by Keith Symon, University of Wisconsin, Marison, WI. 


$$
\frac{\mathrm{dz}}{\mathrm{d} \vartheta}=\frac{\mathrm{rB}_{\mathrm{z}}(\mathrm{r}, z, \vartheta)}{\mathrm{B}_{\vartheta}(\mathrm{r}, \mathrm{z}, \vartheta)}
$$

In general, these equations are not in Hamiltonian form. However, we can try to find a coordinate transformation to variables $x(r, z, \vartheta), \tau(r, z, \vartheta)$ so that the equations for $\chi, \tau$ are in Hamiltonian form. Note that the equations for $\chi, \tau$ are

$$
\begin{aligned}
& \frac{\mathrm{d} \chi}{\mathrm{d} \vartheta}=\frac{\mathrm{rB}}{\mathrm{B}_{\vartheta}} \frac{\partial \chi}{\partial \mathrm{r}}+\frac{\mathrm{rB}_{\mathrm{z}}}{\mathrm{B}_{\vartheta}} \frac{\partial \chi}{\partial z}+\frac{\partial \chi}{\partial \vartheta}, \\
& \frac{\mathrm{d} \tau}{\mathrm{d} \vartheta}=\frac{r \mathrm{~B}_{\mathrm{r}}}{\mathrm{B}_{\vartheta}} \frac{\partial \tau}{\partial \mathrm{r}}+\frac{\mathrm{rB}_{z}}{\mathrm{~B}_{\vartheta}} \frac{\partial \tau}{\partial z}+\frac{\partial \tau}{\partial \vartheta} .
\end{aligned}
$$

These equations are in Hamiltanian form if

$$
\begin{aligned}
& \frac{\mathrm{d} \chi}{\mathrm{d} \vartheta}=\frac{\partial \mathrm{H}(\chi, \tau, \vartheta)}{\partial \tau}, \\
& \frac{\mathrm{d} \tau}{\mathrm{d} \vartheta}=-\frac{\partial H(\chi, \tau, \vartheta)}{\partial \chi}
\end{aligned}
$$

A necessary and sufficient condition for this to be true locally is that

$$
\frac{\partial}{\partial \chi}\left(\frac{d \chi}{d \vartheta}\right)+\frac{\partial}{\partial \tau}\left(\frac{d \tau}{d \vartheta}\right)=0
$$

In the appendix it is shown that Eq. (7) is satisfied if

$$
\operatorname{det}\left(\begin{array}{ll}
\frac{\partial \chi}{\partial r} & \frac{\partial \chi}{\partial z} \\
\frac{\partial \tau}{\partial r} & \frac{\partial \tau}{\partial z}
\end{array}\right)=B_{\vartheta}(r, z, \vartheta)
$$


If this is true, then the equality of the right-hand sides of Eqs. (3) and (5) is the same equation tiat results from the equality of the right-hand sides of Eqs. (4) and (6); i.e., a solution of one automatically satisfies the other. We set the right-hand side of Eq. (3) equal to the right-hand side of Eq. (5) and find

$$
\frac{\partial \mathrm{H}}{\partial \tau}=\frac{r \mathrm{~B}_{\mathrm{r}}}{\mathrm{B}_{\vartheta}} \frac{\partial \chi}{\partial \mathrm{r}}+\frac{\mathrm{rB}_{z}}{\mathrm{~B}_{\vartheta}} \frac{\partial \chi}{\partial z}+\frac{\partial \chi}{\partial \vartheta}
$$

Now,

$$
\frac{\partial \mathrm{H}}{\partial \tau}=\frac{\partial \mathrm{r}}{\partial \tau} \frac{\partial \mathrm{H}}{\partial \mathrm{r}}+\frac{\partial \mathrm{z}}{\partial \tau} \frac{\partial \mathrm{H}}{\partial \mathrm{z}}
$$

and in the appendix it is shown that

$$
\frac{\partial r}{\partial \tau}=-\frac{1}{\Sigma_{v}} \frac{\partial \chi}{\partial z}
$$

$$
\frac{\partial z}{\partial \tau}=\frac{1}{B_{\vartheta}} \frac{\partial x}{\partial r}
$$

so Eq. (9) becomes

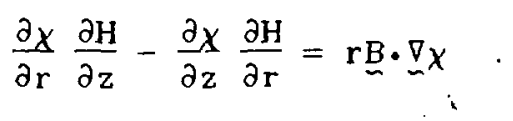

It is not obvious that Eq. (13) has a global solution. However, let us choose

$$
x=\mathrm{r}
$$


We then see that Eq. (8) implies

$$
\tau=\int_{0}^{z} \mathrm{~d} z \mathrm{~B}_{\vartheta}\left(\mathrm{r}, \mathrm{z}^{\prime}, \vartheta\right)
$$

Equations (14) and (15) give the coordinate transformation; it is clearly globally defined. With this choice Eq. (13) becomes

$$
\frac{\partial H}{\partial z}=r B_{r}(r, z, v)
$$

with the obvious solution

$$
H=r \int_{0}^{z} B_{r}\left(r, z^{\prime}, \vartheta\right) d z^{\prime}
$$

Note that in Eq. (17) $H$ is given as a function of $r, z$. To express $H$ as a function of canonical variables, we must invert the transformation of Eqs. (14) and (15). The Hamiltonian is clearly globally defined.

\section{REFERENCES}

1. V. I. Arnold and A. Avez, Ergodic Problems of Classicel Mechanics (W. A. Benjamin, Inc., New York, 1968), pp. 81-114.

2. E. Zehnder, "Homoclinic Points Near Elliptic Fixed Points," Comm. Pure Apfl. Math. XXVI, 131-182 (1973). 
Using Eqs. (3) (4), (A-1), (A-2), (A-5), (A-6), (A-7), and $(A-8)$ in Eq. (7), we find

$$
\begin{aligned}
& \frac{\partial^{2} \chi}{\partial \mathrm{r}^{2}}\left(\frac{\partial \tau}{\partial z} \frac{\mathrm{rB}_{\mathrm{r}}}{\mathrm{B}_{\vartheta}}\right)+\frac{\partial^{2} \chi}{\partial \mathrm{r} \partial \mathrm{z}}\left(\frac{\partial \tau}{\partial z} \frac{\mathrm{rB}_{z}}{\mathrm{~B}_{\vartheta}}-\frac{\partial \tau}{\partial \mathrm{r}} \frac{\mathrm{rB}_{\mathrm{r}}}{\mathrm{B}_{\vartheta}}\right) \\
& +\frac{\partial^{2} \chi}{\partial \mathrm{r} \partial \vartheta} \frac{\partial \tau}{\partial \mathrm{z}}-\frac{\partial^{2} \chi}{\partial \mathrm{z} \partial \vartheta} \frac{\partial \tau}{\partial \mathrm{r}}-\frac{\partial^{2} \chi}{\partial \mathrm{z}^{2}}\left(\frac{\partial \tau}{\partial \mathrm{r}} \frac{\mathrm{rB}_{\mathrm{z}}}{\mathrm{B}_{\vartheta}}\right) \\
& -\frac{\partial^{2} \tau}{\partial r^{2}} \frac{\partial \chi}{\partial z} \frac{r B_{r}}{B_{\vartheta}}+\frac{\partial^{2} \tau}{\partial r \partial z}\left(-\frac{\partial \chi}{\partial z} \frac{r B_{z}}{B_{\vartheta}}+\frac{\partial \chi}{\partial r} \frac{r B_{r}}{B_{\vartheta}}\right) \\
& -\frac{\partial^{2} \tau}{\partial \mathrm{r} \partial \vartheta} \frac{\partial \chi}{\partial z}+\frac{\partial^{2} \tau}{\partial z \partial \vartheta} \frac{\partial \chi}{\partial \mathrm{r}}+\frac{\partial^{2} \tau}{\partial z^{2}} \frac{\partial \chi}{\partial \mathrm{r}} \frac{\mathrm{rB}_{2}}{\mathrm{~B}_{\vartheta}} \\
& \left.+\left(\frac{\partial \chi}{\partial r} \frac{\partial \tau}{\partial z}-\frac{\partial \chi}{\partial z} \frac{\partial \tau}{\partial r}\right) \mid \frac{\partial}{\partial r}\left(\frac{\mathrm{rB}_{\mathrm{r}}}{\mathrm{B}_{\vartheta}}\right)+\frac{\partial}{\partial z}\left(\frac{\mathrm{rB}_{2}}{\mathrm{~B}_{\hat{\tau}}}\right)\right]=0 \quad . \quad(\mathrm{A}-9)
\end{aligned}
$$

If, in Eq. (A-9), we use

$$
\underline{\nabla} \cdot \underline{B}=0
$$

and rearrange terms, we obtain

$$
\underline{B} \cdot \underset{\sim}{\nabla}(\ln J)=\underline{B} \cdot \underline{\nabla}\left(\ln B_{\vartheta}\right)
$$

One solution of this equation is clearly

$$
\mathbf{J}=\mathrm{B}_{\vartheta} .
$$

Note that Eqs. (A-12), (A-6), and (A-7) together imply Eqs. (11) and (12). 


\section{DERIVATION OF HAMILTONIAN CONDITION}

We mist show that Eq. (7) is satisfied if Eq. (8) is. First, observe that

$$
\frac{\partial}{\partial \chi}=\left(\frac{\partial r}{\partial \chi} \frac{\partial}{\partial r}+\frac{\partial z}{\partial \chi} \frac{\partial}{\partial z}\right)
$$

$$
\frac{\partial}{\partial \tau}=\left(\frac{\partial r}{\partial \tau} \frac{\partial}{\partial r}+\frac{\partial z}{\partial \tau} \frac{\partial}{\partial z}\right)
$$

Furthermore, we know that

$$
\left(\begin{array}{ll}
\frac{\partial r}{\partial \chi} & \frac{\partial r}{\partial \tau} \\
\frac{\partial z}{\partial \chi} & \frac{\partial z}{\partial \tau}
\end{array}\right)\left(\begin{array}{ll}
\frac{\partial \chi}{\partial r} & \frac{\partial \chi}{\partial z} \\
\frac{\partial \tau}{\partial r} & \frac{\partial \tau}{\partial z}
\end{array}\right)=\left(\begin{array}{ll}
1 & 0 \\
0 & 1
\end{array}\right) .
$$

Thus, if we let

$$
J \equiv \operatorname{det}\left(\begin{array}{cc}
\frac{\partial x}{\partial r} & \frac{\partial x}{\partial z} \\
\frac{\partial \tau}{\partial r} & \frac{\partial \tau}{\partial z}
\end{array}\right)
$$

then

$$
\begin{aligned}
& \frac{\partial r}{\partial \chi}=\frac{1}{J} \frac{\partial \tau}{\partial z} \\
& \frac{\partial r}{\partial \tau}=-\frac{1}{J} \frac{\partial \chi}{\partial z} \\
& \frac{\partial z}{\partial \tau}=\frac{1}{J} \frac{\partial x}{\partial r} \\
& \frac{\partial z}{\partial \chi}=-\frac{1}{J} \frac{\partial \tau}{\partial r}
\end{aligned}
$$

